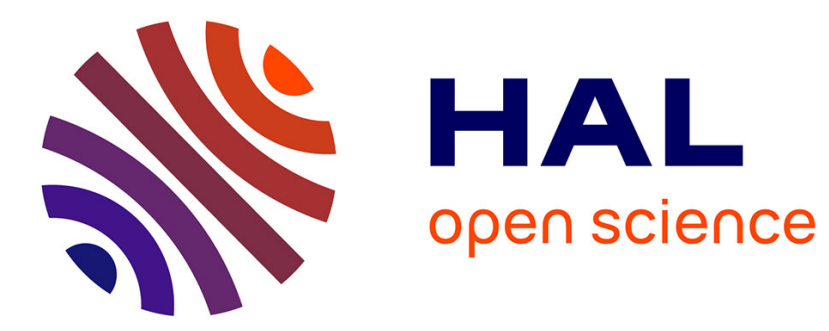

\title{
Mechanism-based approach for the deployment of a tensegrity-ring module
}

Landolf Rhode-Barbarigos, C. Schulin, Nizar Bel Hadj Ali, René Motro, Ian F.C. Smith

\section{- To cite this version:}

Landolf Rhode-Barbarigos, C. Schulin, Nizar Bel Hadj Ali, René Motro, Ian F.C. Smith. Mechanismbased approach for the deployment of a tensegrity-ring module. Journal of Structural Engineering, 2012, 138 (4), pp.539-548. 10.1061/(ASCE)ST.1943-541X.0000491 . hal-00855809

\section{HAL Id: hal-00855809 \\ https://hal.science/hal-00855809}

Submitted on 30 Aug 2013

HAL is a multi-disciplinary open access archive for the deposit and dissemination of scientific research documents, whether they are published or not. The documents may come from teaching and research institutions in France or abroad, or from public or private research centers.
L'archive ouverte pluridisciplinaire HAL, est destinée au dépôt et à la diffusion de documents scientifiques de niveau recherche, publiés ou non, émanant des établissements d'enseignement et de recherche français ou étrangers, des laboratoires publics ou privés. 


\title{
Mechanism-based approach for the deployment of a tensegrity- ring module
}

\author{
L. Rhode-Barbarigos ${ }^{1}$, C. Schulin ${ }^{2}$, N. Bel Hadj Ali ${ }^{3}$, R. Motro ${ }^{4}$, I.F.C. Smith ${ }^{5}$
}

\begin{abstract}
Tensegrity structures are spatial systems composed of tension and compression components in a self-equilibrated prestress stable state. Although the concept is over 60 years old, few tensegrity-based structures have been used for engineering purposes. Tensegrity-ring modules are deployable modules composed of a single strut circuit that, when combined, create a hollow rope. The "hollow-rope" concept was shown to be a viable system for a tensegrity footbridge. This paper focuses on the deployment of pentagonal ring modules for a deployable footbridge application. The deployment sequence of a module is controlled by adjusting cable lengths (cable actuation). The geometric study of the deployment for a single module identified the path space allowing deployment without strut contact. Additionally, a deployment path that reduces the number of actuated cables was found. The number of actuated cables is further reduced by employing continuous cables. A first generation prototype was used to verify experimentally both findings. The structural response during both unfolding and folding is studied numerically using the dynamic relaxation method. The deployment-analysis algorithm applies cable-length changes first to create finite mechanisms allowing deployment and then to find new equilibrium configurations. Therefore, the actuation-step size is identified as the most critical parameter for a successful deployment analysis. Finally, it is shown that the deployability of the footbridge does not affect its element sizing since stresses during deployment are lower than in-service values.
\end{abstract}

\section{Introduction}

Tensegrity structures are structural systems that are composed of tension and compression components in a self-equilibrated pretension stable state. Scientists and engineers in fields such as bio-engineering, aerospace engineering, robotics, architecture and civil engineering have studied these structures (Motro 2005; Skelton and de Oliveira 2009). In bio-engineering, the tensegrity concept is used to model biological systems such as cytoskeleton structures of unicellular organisms (Ingber 1998; Volokh et al. 2000). In aerospace technology, the concept offers an alternative solution to design lightweight deployable structures such as masts, solar arrays and antennas (Furuya 1992; Tibert 2002). Furthermore, tensegrity has also inspired research on string-driven robotic systems (Aldrich and Skelton 2003; Graells Rovira and Mirats Tur 2009). Architects and engineers are investigating responsive architecture and the use of tensegrity systems in adaptive buildings (Adam and Smith 2007a; Aldrich and Skelton 2003; d'Estrée 2003). Tensegrity structures are composed of axially loaded loaded structural elements, cables and struts. Mass in tensegrity structures can thus be used in a more efficient way compared with systems having orthogonal material topologies (Skelton and de Oliveira

\footnotetext{
${ }^{1}$ Doctoral Assistant, IMAC, Ecole Polytechnique Fédérale de Lausanne, Lausanne, Switzerland (corresponding author). Email: landolf-giosef.rhode-barbarigos@epfl.ch

${ }^{2}$ Market Analyst, Holcim Group Support Ltd., Holderbank, Switzerland.

${ }^{3}$ Assistant Professor, LASMAP, Ecole Polytechnique de Tunisie, University of Carthage, La Marsa, Tunisia.

${ }^{4}$ Professor Emeritus, LMGC, Université de Montpellier, Montpellier, France.

${ }^{5}$ Professor, IMAC, Ecole Polytechnique Fédérale de Lausanne, Lausanne, Switzerland.
} 
2009). Therefore, tensegrity structures have a high strength to mass ratio that provides the possibility of designing strong and lightweight structures. Tensegrity systems are also particularly attractive for active and deployable structures since the amount of energy needed for control and adaptation is often smaller than that required for other structural systems (Skelton et al. 2009).

Active structures are structures having the ability to change their shape and behavior in response to changes in their environment. Due to integrated actuated elements, active structures are capable of interacting with complex environments. Fest et al. (2004) experimentally explored the potential of a five-module tensegrity structure with telescopic struts. Advanced computing techniques such as stochastic search, case-based reasoning and reinforcement learning have been successfully used to demonstrate biomimetic properties of active tensegrity structures (Adam and Smith 2008; Domer 2003; Domer and Smith 2005). These methods enable active tensegrity structures to learn from previous control experience, thereby providing improved service performance. Tensegrity systems can also be faulttolerant. A single damaged member does not necessarily lead to structural collapse. Computational approaches that support self-diagnosis and self-repair have also been developed (Adam and Smith 2007b). Furthermore, dynamic behavior and vibration control of the five-module active tensegrity structure were studied in order to shift the natural frequencies away from excitation (Bel Hadj Ali and Smith 2010).

Due to their shape-changing ability, deployable structures are attractive candidates for active structures. Deployable structures vary their shape from a compact, packaged configuration to an expanded, operational configuration. Active control can be used to control the deployment path of the structure and to correct the final position in structures such as reflectors or antennas where high precision is required. Research into deployable structures revealed that functionality and feasibility of the design of deployable structures depend not only on the structural behavior of the final configuration under service loads but also on the structural response during deployment (Pellegrino 2001). Therefore, geometrical analyses should be combined with studies of the structural response during both deployment phases: unfolding and folding.

The most common method for deploying structures is the creation of a finite mechanism. This refers to structures that deploy by moving rigid members relative to each other, using hinges for example. Most deployable systems are composed of single degree of freedom deployable units such as pantographic mechanisms or scissor-like elements. Gantes et al. $(1991 ; 1989)$ studied deployable structures having scissor-like elements. Studies revealed nonlinearity during deployment, high sensitivity and unbalanced force distribution (Gantes 2001). Tan and Pellegrino (2008) investigated the nonlinear behavior of a cable-stiffened pantographic deployable structure. Experiments were conducted on a small-scale physical model where deployment is achieved employing actuated cables. Single degree of freedom deployable units require few actuated elements for shape control. If more complex units such as tensegrity modules are used for deployment, then deployment strategies with minimal actuation should be explored.

The application of lightweight tensegrity systems to deployable structures is a natural evolution of the almost sixty-year old concept; there are current research projects worldwide (Fest 2002; Motro 2005; Pellegrino 2001; Skelton and de Oliveira 2009). The concept of deployable tensegrity structures first appeared in 1990s. Furuya (1992) investigated deployment approaches of a tensegrity mast from geometrical viewpoint. Hanaor (1993) performed the deployment of a simplex-based tensegrity grid using telescopic struts. Bouderbala and Motro (1998) studied the folding of expandable octahedron assemblies and showed that cable mode folding was less complex than strut mode, although the latter produced a more compact package. Sultan and Skelton (2003) proposed a cable-control 
deployment strategy for tensegrity structures based on the existence of an equilibrium manifold. Assuming that all cables are controlled, actuation is conducted such that the structure remains close to a stable equilibrium throughout the deployment. Pinaud et al. (2004) implemented cable-control deployment of a small-scale tensegrity boom composed of two tensegrity modules and studied asymmetrical reconfigurations during deployment. Smaili and Motro (2005) investigated folding of tensegrity systems by activating finite mechanisms. A cable-control strategy was applied to a double layer tensegrity grid. The proposed strategy was then extended to the folding of curved tensegrity grids. Similarly, Sultan (2009) presented a shape control strategy for tensegrity structures in which the motion is controlled through infinitesimal mechanism directions. In these cases, cable actuation was used to control deployment. Although most of the studies focus on a controlled deployment motion, they do not take into account loading and do not explore actuation strategies that use continuous cables.

Cable-control deployment can be used to direct the tensegrity structure to maintain its stiffness as it moves from one equilibrium position to another (Sultan and Skelton 2003) or to follow particular paths based on the nonlinear equations of motion (Sultan et al. 2002). There are, however, disadvantages with this actuation strategy. Tibert and Pellegrino (2003) argued that controlling cables is complicated, because many additional mechanical devices are necessary. Instead of using cables they proposed deployment with foldable struts, for which self-locking tape spring hinges were used. They also experimentally investigated the use of telescopic struts for the deployment of tensegrity reflectors (Tibert 2002). A disadvantage of this foldable/telescopic strut strategy is that the structure has no stiffness until it is fully deployed. In their studies, Tibert and Pellegrino (2003) compared the stiffness of a deployable tensegrity mast with a conventional mast. They identified lack of stiffness during deployment and weak deployed bending stiffness as obstacles to practical applications for the studied topology. However, in this module topology the absence of continuous compression members is the cause of low bending stiffness and not the presence of internal mechanisms. Deployment can benefit from the application of zero-free-length springs. Schenk et al. (2007) studied the application of zero-free-length springs in tensegrity systems transforming tensegrity structures to statically balanced mechanisms. Le Saux et al. (2004) studied the problem of collisions between bars during deployment. Motro et al. (2006) proposed a family of deployable cable-controlled tensegrity modules called "tensegrity rings" that can be assembled in a "hollow rope". Although tensegrity rings are composed of a single strut circuit, their deployment can be completed without strut collision. The "hollow rope" concept shows promise for architecture and civil engineering applications such as pedestrian bridges (Rhode-Barbarigos et al. 2010b; Rhode-Barbarigos et al. 2010a). However, its application for a deployable footbridge has not been explored.

This paper focuses on the deployment of pentagonal tensegrity-ring modules that are viable for a deployable tensegrity footbridge. The deployment of a bridge module is analyzed first geometrically and then numerically under dead load. A geometric study of a single bridge module is carried out in order to investigate the path space allowing deployment without strut contact. A deployment path reducing the number of actuated cables is identified. Furthermore, continuous cables are integrated in ring-module topology to further reduce the number of actuated cables required. Both cases were also studied experimentally on a small scale prototype. Finally, the structural response of the bridge module during deployment is analyzed using an algorithm based on the dynamic relaxation method. Quasi-static actuation and frictionless motions are assumed in this study.

\section{Tensegrity-ring modules and footbridge application}


The topology of the module studied in this paper belongs to a special family of tensegrity modules classified by previous work as "ring modules" (Motro et al. 2006). Tensegrity-ring module topology was first presented by Pugh (1976). Struts in tensegrity-ring modules are joined one to another creating a single circuit with an empty space in the middle. Due to strutto-strut connectivity, ring modules are classified as class II tensegrity systems (Skelton and de Oliveira 2009). Ring modules are named after the polygon that describes the end of the ring. The deployability of ring modules along with a new construction method were studied by Motro (2006) showing that tensegrity-ring topology assures deployability. It was also found that the deployment can be controlled by changing the rest length of the cables. However, cable-length changes for a contact free deployment were not explored. Nguyen (2009) studied the structural behavior of the deployed pentagonal ring module under compression, tension and bending. He showed that cable stiffness is more important for the overall stiffness of the module compared with strut stiffness. A rheological model based on a series of two springs was proposed. The folding of the pentagonal module was studied using FEM. Nodal displacements were applied while cables were removed or attributed with low values of Young modulus. Although this method provides a similar deployment motion, it is not suitable for studying actuation strategies and the structural response of the module.

In a footbridge application, ring modules are elementary components interconnected according to the "hollow rope" concept (Motro et al. 2006). Their internal empty ring space is used as walking space with the addition of a deck. Previous work by the authors revealed that tensegrity-ring modules are viable structural systems for a footbridge application (Bel Hadj Ali et al. 2010; Rhode-Barbarigos et al. 2010). Based on the deployability of ring modules, the structural system of the tensegrity-ring bridge can be extended for a deployable footbridge application. Cases of fast erection or space constraints can benefit from the use of a deployable structural system. In this study, deployment is assumed over a $16 \mathrm{~m}$ navigable waterway with the bridge deploying from both sides and joining in the middle. Each part of the bridge is composed of two identical tensegrity modules with a $4 \mathrm{~m}$ span (Figure 1). During the deployment, boundary conditions allow all nodal movements required for unfolding and folding. However, nodes at both ends of the bridge are blocked during service assuring the required stiffness for the structural system. Furthermore, a foldable deck made of sliding plates is also assumed. The deployment aspects of a pentagonal tensegrity-ring module, constitutive structural system of the bridge, are addressed in this study.

Among tensegrity-ring modules, the pentagonal ring topology has the minimum number of struts that assure a smooth deployment. Moreover, the study of the kinematic and static properties of pre-stressed ring modules revealed that the pentagon module has no infinitesimal mechanisms and six independent states of self-stress. Furthermore, the pentagonal ring module was found to be structurally the most efficient module for the tensegrity footbridge application based on a structural efficiency index (Rhode-Barbarigos et al. 2010a). Therefore, the pentagonal ring module was chosen as the elementary module for the deployable tensegrity bridge.

The pentagonal ring-module topology can be described with straight prism geometry (Figure 2). Considering a straight pentagonal prism, the nodes of its two pentagonal faces are connected with cables called layer cables. Two of the nodes on each lateral side of the polygon are connected by a diagonal strut. Layer cables and diagonal struts are shown in Figure 2a. The two pentagonal faces are also connected by a pair of struts starting at the second left node of one side and ending at the first right node of the second following side (Figure $2 b$ ). Figure $2 c$ shows the strut circuit for a single pentagonal module highlighting the five pairs of intermediate struts. The rotational direction of intermediate strut pairs is inversed compared with the direction of diagonal struts. The rotational order must be respected for both strut families in order to obtain a single circuit. Finally, the middle node of each pair of 
intermediate struts is connected to every node on the same lateral side with cables. Figure $2 \mathrm{~d}$ shows the cable connections for a single strut pair. These cables are named $x$-cables inspired from their " $\mathrm{x}$ " form on each prism side. Based on their direction, $\mathrm{x}$-cables can be further classified on coplanar $x$-cables and non-coplanar $x$-cables in relation to the diagonal strut on the same side. Figure 2e shows all the cables for a single module including 10 layer cables and $20 \mathrm{x}$-cables. Finally, Figure $2 \mathrm{f}$ shows the entire pentagonal ring module. In ring module topology, strut lengths may differ among diagonal struts and the intermediate paired struts. However, in this study the pentagonal module topology maintains a unique strut length.

Pentagonal ring modules like all tensegrity systems are stable systems. Therefore, the deployment of the pentagonal ring module is only possible if cable lengths are controlled. Allowing cables to change lengths permits strut movement during unfolding and folding. Previous studies (Motro et al. 2006) showed that during unfolding the area of the two pentagonal faces of the straight prism decreases while the area of its lateral sides increases. Consequently, during unfolding layer cable length decreases while x-cable length increases. The inverse occurs during folding.

\section{Geometric study of deployment}

During deployment, the geometry of the structure changes from a compact configuration to an expanded one. Therefore, a geometric study of the structure is the starting point for any deployment analysis. Most of deployable structures are usually composed of deployable single-degree-of-freedom units. Consequently, their deployment can be easily described by a single motion, such as a translation or a rotation. The pentagonal tensegrity-ring module is a stable spatial system. Therefore, in order to allow deployment, cable lengths must be varied resulting in a multiple-degree-of-freedom mechanism. Its deployment can be described with a minimum of three motions: translation, rotation and dilation. The first motion is the translation of the two pentagonal faces on the longitudinal axis of the module. The distance between the two faces defines the length of the module which increases during unfolding. In order to position the strut circuit inside a new module length, a transverse rotation of the pentagonal faces is required. Finally, the third motion is the radial dilation of the pentagonal faces. Both requirements can be easily understood by following the trajectory of diagonal struts under two module lengths. Diagonal struts rotate on both horizontal and vertical planes in order to fit in the new module length.

The geometry of the ring module can therefore be described by three parameters: the module length $L$, the radius of the circumscribed circles on the pentagonal faces $R$ and the angle measuring the transverse rotation among the two pentagonal faces $\theta$. Figure 3 shows the three parameters in relation with the pentagonal straight prism geometry. The module length $L$ takes the minimum value $L_{\min }$ for the folded length and increases with unfolding until reaching the maximum value $L_{\max }$ for the deployed configuration. On the contrary, the radius $R$ has the maximum value $R_{\max }$ for the folded length and decreases until a minimum value $R_{\min }$ for the unfolded configuration. Finally, the value of the transverse rotation $\theta$ varies from a maximum value $\theta_{\max }$ for the folded configuration to zero for the unfolded configuration. Table 1 gives the nodal cylindrical and Cartesian coordinates of the pentagonal ring-module topology based on $L, R$ and $\theta$. Node connectivity is given in Appendix. Strut and cable lengths can be estimated based on these three parameters.

The relationship between the parameters $L, R$ and $\theta$ cannot be explicitly formulated as it depends on the deployment path chosen. There are many paths that can lead to the same unfolded length. However, choosing a path where parallelism of the pentagonal faces is respected provides better deployability. Moreover, this deployment path will be smooth without any element contact avoiding local instabilities and dynamic effects due to strut congestion. Furthermore, if dead load is applied the risk of strut contact increases due to the 
deflection of the structure. Consequently, the path chosen should have enough space between each couple of struts to avoid contact. Another important criterion is the number of actuated cables for deployment. The more cables need to change length during deployment, the more complicated the design of the structure becomes due to actuation equipment. In addition, increasing the number of actuated cables increases the complexity of control during deployment. Other criteria such as path robustness, energy requirements and cost are not part of this study.

If the deployment path respects the module topology then the parameters that are necessary for a geometrical description are decreased to only two: the length of the module $L$ and the transverse rotation between the pentagonal faces of the module $\theta$. Consequently, the deployment-path space becomes two-dimensional and can be found by computing all couples of $L$ and $\theta$ values for which the struts do not interfere. Figure 4 shows the deployment-path space for the pentagonal bridge module with a deployed length of $400 \mathrm{~cm}$ (bridge span of 16 $\mathrm{m})$. The allowable path space is shown in white. The most compact configuration is obtained with a transverse rotation of $0.37 \mathrm{rad}\left(21.2^{\circ}\right)$ and has a folded length of $60 \mathrm{~cm}$. If no rotation is allowed, the compact configuration is limited to $373 \mathrm{~cm}$ before some of the struts start touching. Hence to efficiently unfold and fold the structure, both translation and rotation of the pentagonal faces are necessary. The isometric curves in Figure 4 represent the closest distance between struts from 5 (inner curve) to $45 \mathrm{~cm}$ (outer curve). The tip of each isometric curve is used to define the path along which the distance between the struts is maximized and consequently the risk of strut contact is minimized. The same path is followed for both unfolding and folding. However, this path is not an optimal one as it requires the actuation of all cables in a single module. Additionally, the desired deployed module length of $400 \mathrm{~cm}$ is exceeded.

The feasibility of the concept including deployment motions and extreme values of the deployment-path space can be easily validated experimentally on an elementary small-scale physical model of the pentagonal tensegrity-ring module. Figure 5 shows the physical model and its corresponding theoretical path space with isometric curves varying from 1 to $9 \mathrm{~cm}$. The model is made out of wooden struts and elastic rope. Using an elastic rope allows cables to adjust their length according to the module length and therefore provides deployability for the model. The deployment is conducted manually either by applying forces on the nodes of one of the pentagonal faces or by changing the length of the corresponding cables. The fully deployed model has a length of approximately $75 \mathrm{~cm}$ with a radius of $57 \mathrm{~cm}$. All struts have a diameter of $3 \mathrm{~cm}$ and a length of $100 \mathrm{~cm}$. Cable length at the fully deployed position is $67 \mathrm{~cm}$ and $52 \mathrm{~cm}$ for layer cables and x-cables respectively. Nested steel hooks were used for the strut-to-strut connection. Steel hooks are convenient for the construction of the model. However, they induce eccentricities and this means that the accuracy of the numerical model is affected. Finally, individual segments of elastic rope are fixed on each hook for each cable of the module. Based on this physical model, extreme values of $L$ and $\theta$ in the deploymentpath space (strut contact) were determined. Starting from a deployed configuration, the deployment path measured on the physical model deviates from the theoretical deployment path with folding due to use of steel hooks in joint design. Consequently, the folded length is slightly increased. However, full reversible deployment (unfolding and folding) was achieved without strut contact, thus validating the feasibility of the concept.

\section{Actuation for deployment}

The main requirement for the deployability of tensegrity-ring modules is that cables change length and therefore, actuation is required. Cable actuation can be obtained with the use of pulleys and automated cranks adjusting cable length to the required length. Ring modules in 
their initial expanded configuration are stable systems. If cable length can be adjusted, then ring modules become multiple-degrees-of-freedom mechanisms allowing strut movement for unfolding and folding. Both cable families of the pentagonal ring module (layer cables and $\mathrm{x}$ cables) should be actuated for deployment resulting to 30 actuators for a single pentagonal ring module: 10 for layer cables and 20 for x-cables. Based on this actuation strategy, each part of the deployable tensegrity footbridge (incl. two interconnected modules) requires 55 actuators: 15 on layer cables and 40 on $\mathrm{x}$-cables. The maximum number of actuators required for the entire bridge is thus 110 .

A deployment path requiring a lower number of actuators was found based on observations on the path with the minimal risk of strut contact. Along this new path, the length of the coplanar $\mathrm{x}$-cables is kept constant throughout unfolding and folding. Although coplanar $\mathrm{x}$-cables are not actuated, they remain in tension during both unfolding and folding. Figure 6 shows the two deployment paths for the bridge module inside the corresponding deployment-path space. Both paths are valid for both deployment phases (unfolding and folding). The curve describing the path with minimum number of actuators is similar to the curve of the path with the minimal contact risk but with a lower relative rotation among the two pentagonal faces. Moreover, this path ends at the desired deployed length. The folded length of the module is slightly increased and it may increase more if dead load is taken into consideration. Nevertheless, the increment in the folded length is not important compared with the decrease in the number of actuators required: 20 actuators for a single module and 35 for each part of the deployable tensegrity bridge (70 actuators for the entire bridge). Another important characteristic of this path is that layer cables and non-coplanar $\mathrm{x}$-cables have clear functions during unfolding and folding. Layer cable action controls unfolding while x-cable action controls folding. Consequently, layer cables are shortened for unfolding while x-cables are lengthened in order to ensure that the defined path is followed. The inversed scenario is followed during folding. Moreover, combined action on both layer cables and x-cables can assure that there is enough rigidity in the structure during the deployment manifold.

In order to reduce further the number of actuators, continuous cables are used in the module. However, the effect of continuous cables on the structural behavior of the tensegrity-ring module requires examination as they reduce the number of independent states of self-stress and the number of internal mechanisms in the module (Bel Hadj Ali et al. 2011; Moored and Bart-Smith 2009). For deployment, continuous cables can only replace cables of the same family since deployment action should be the same. Furthermore, it is desirable to have continuous cables without abrupt changes of direction in order to avoid additional non-axial forces in the struts of the module. Consequently, continuous cables may be applied for layer cables, coplanar x-cables and non-coplanar x-cables separately. Thus, the final continuous cable configuration of the pentagonal ring module includes 2 continuous layer cables, 5 continuous coplanar x-cables and 5 continuous non-coplanar $x$-cables reducing the number of actuators for a single module from 30 to 12 . Unfortunately, this configuration is unstable and therefore, the application of continuous cables is limited to x-cables only.

If the continuous cable module follows the deployment path with fixed coplanar $\mathrm{x}$-cable length then actuation in coplanar $\mathrm{x}$-cables is no longer required. Consequently, cable continuity in coplanar $\mathrm{x}$-cables is not necessary. In this case, the deployment can be achieved with minimum number of actuators as only 15 actuators are needed for a single module. The decrement in the number of actuators required is more important in the case of the deployable tensegrity footbridge considering continuous non-coplanar $\mathrm{x}$-cables that run on both interconnected modules. For each part of the tensegrity bridge (two interconnected modules), only 20 actuators are required for deployment: 15 for layer cables and 5 for the continuous non-coplanar $\mathrm{x}$-cables. The total number of actuators required for the entire bridge is thus reduced to 40 (initial number: 110). 
The use of continuous cables and its effect on the deployment was verified experimentally on the elementary physical model of the tensegrity pentagonal ring module. The deployment path with constant length for coplanar x-cables was equally validated experimentally for the continuous module. For the validation of this path, the elastic rope segments of coplanar $\mathrm{x}$ cables were replaced by non-extensible nylon rope segments. Cable continuity was not used in coplanar x-cables as their length remains constant. Figure 7 shows the small-scale physical model with continuous cables in its folded and unfolded configurations. A full deployment of the model was successfully conducted without any strut contact as the use of continuous cables does not modify the desired deployment motion.

\section{Structural response during deployment}

The geometrical study resulted in a contact free deployment path with minimal actuation. However, the structural response of the module during deployment was not considered. Consequently, in order to assure that there is no risk of failure during deployment, the structural response of the pentagonal ring module is studied throughout unfolding and folding. During both phases, dead load is the only loading applied in the structure. In this study, quasistatic actuation and frictionless motion are assumed for deployment. Quasi-static actuation guarantees that acceleration and velocity terms in the deployment analysis can be neglected. Therefore, the dynamic analysis of the deployment is approximated by a static analysis. The static analysis of the pentagonal tensegrity-ring module is conducted using the dynamic relaxation method, a well known static method that is suitable for non-linear structures. Dynamic relaxation follows the response of the structure from the moment of loading until reaching equilibrium due to fictitious masses and kinetic damping. Hence, this method only analyzes "snapshots" of the deployment. A modified version of the dynamic relaxation method for continuous cables is used in this study (Bel Hadj Ali et al. 2011). This version exploits that the governing equations for a tensegrity structure with continuous cables are related to those of an equivalent "classic" tensegrity (without continuous cables). Therefore, the modified version is suitable for the static analysis of the continuous cable configuration of the pentagonal ring module.

An analysis algorithm was conceived for deployment of tensegrity modules. This algorithm is based on the actuation required for the deployment and uses the modified version of the dynamic relaxation method. Module topology and element characteristics are given as input for the analysis. The algorithm provides a description of the deployment path under loading and an illustration of the deployment movement. Internal forces are displayed independently for all elements throughout unfolding and folding. A deployment path can be predefined in the algorithm. Deployment actuation is implemented as an increase or decrease of element length. Actuated elements and actuation steps are defined using analyses of the tensegritymodule and the desired deployment path followed. Furthermore, actuation steps may vary for every actuated cable. After each actuation phase a new equilibrium is found using the dynamic relaxation method as shown in Figure 8. Apart from the constraint to avoid strut contact, the algorithm includes also a constraint for internal forces. The deployment involves the creation of finite mechanisms allowing the module to change length. Additional constraints may be required in cases where the dead load affects considerably the geometry of the module during deployment.

In the case of the pentagonal ring module, the deployment analysis can start from the folded or the unfolded configuration. Actuated elements are defined from the cable action required for deployment and the path chosen. An overview of the deployment analysis algorithm for the pentagonal ring module is presented in Figure 8. In order to create a finite mechanism for unfolding, non-coplanar $\mathrm{x}$-cables are actuated first. A single actuation step is applied for all non-coplanar $\mathrm{x}$-cables. Increasing the length of actuated $\mathrm{x}$-cables allows the strut circuit to 
change length. Layer cable length is then iteratively decreased until a new equilibrium is found with the dynamic relaxation method. A single actuation step is applied also for all layer cables. The module in the new equilibrium configuration has an increased length. Therefore, repeating this action leads to unfolding the module. Due to the creation of finite mechanisms internal forces remain low from one configuration to another. A constraint is implemented for internal forces. If the increment in internal forces is important, the unfolding phase stops and non-coplanar $\mathrm{x}$-cable length is increased in order to create a new mechanism. The unfolding phase will also stop if the module reaches a predefined unfolded length or strut contact is observed. Hence, every new configuration is constrained by extreme values of module length, strut contact and low internal forces. In the case of the tensegrity bridge, the pentagonal ring module deploys horizontally having dead load vertically to the axis of movement. Consequently, a geometrical control of the planarity of the pentagonal faces is implemented to assure that no excessive deflection is recorded. If the module reaches the predefined unfolded length or contact is observed, then deployment actions are inversed in order to fold the structure.

The deployment analysis algorithm provides a description of the deployment path under loading and the evolution of internal forces in struts and cables throughout unfolding and folding. Figure 9 shows the deployment path for the bridge module topology obtained by the deployment-analysis algorithm. The bridge module includes steel struts with a length of 542 $\mathrm{cm}$ and cables with $277 \mathrm{~cm}$ and $366 \mathrm{~cm}$ for x-cables and layer cables respectively. Furthermore, struts have an external diameter of $11.4 \mathrm{~cm}$ and a $0.6 \mathrm{~cm}$ thickness. Cables have a cross-section area of $1.76 \mathrm{~cm}^{2}$. The analysis is conducted taking into account element dead load as deployment is a service phase. Unfolding and folding follow the same path just like in the geometrical analysis (Figure 9). However, starting from the deployed configuration the path obtained from the analysis is different compared with the geometrical path with minimum number of actuators. The difference observed is due to the effect of dead load that acts vertically on the deployment axis. The deviation from the theoretical path increases with folding. The folded length is thus larger than predicted: from $60 \mathrm{~cm}$ to $120 \mathrm{~cm}$ for the $400 \mathrm{~cm}$ bridge module.

Figure 10 shows the evolution of internal forces for the bridge module topology composed of steel struts and cables. Although the deployment path is the same for unfolding and folding (see Figure 9), internal forces in the module may vary slightly during the two phases. The difference recorded is a result of the equilibrium configurations found by the algorithm for each phase. Unfolding equilibrium configurations are not exactly the same as folding equilibrium configurations. Such discrepancy arises because the structure may have the same deployment length with a different self-stress state. All elements (cables and struts, actuated and non-actuated) show the same trend in internal forces. Internal forces increase with deployment until reaching a local maximum for a module length of approximately $360 \mathrm{~cm}$ and decrease during the rest of the unfolding. Local extrema and inversed tendencies in internal forces of the module are observed during both unfolding and folding. The highest values of internal forces in the module are observed at fully unfolded length. These values correspond to the self-stress state induced for service which is lost with the creation of finite mechanisms for folding. Internal forces remain lower than service values throughout deployment. Moreover, all cables and struts remain axially loaded as in the service phase. There are thus neither slack cables nor struts under tension during deployment. Consequently, the deployment path with minimum actuation found from the geometrical analysis is suitable for the pentagonal ring module.

\section{Discussion}


The deployment of a single pentagonal ring module was studied first geometrically and then numerically using the deployment-analysis algorithm. The geometric analysis provided the deployment path with the minimum number of actuators. The number of actuators was further reduced with the use of continuous cables. The total number of actuators required for the tensegrity bridge is $63 \%$ lower than the initial configuration (from 110 to 40). However, the number of actuators is still high and should be decreased further.

Actuation and deployment path are taken into account as input in the deployment-analysis algorithm, where cable actuation is applied to find equilibrium configurations under a new length. Therefore, the deployment path obtained by this algorithm is a series of equilibrium configurations offering a great advantage for the deployability of the structure. In case of a problematic deployment, the structure is more likely to return to an equilibrium configuration, thus avoiding instabilities that may induce collapse. Additionally, equilibrium offers the possibility to exploit the rigidity of the tensegrity system at any moment of the deployment. The number of intermediate equilibrium configurations in a deployment sequence depends on the actuation-step size applied. Therefore, actuation-step size is a key parameter for the deployment. Large actuation steps may lead to large deployment steps resulting in stability problems and strut contact while small steps are computationally expensive. Smaller steps result in a larger number of equilibrium configurations and lower actuation energy requirements. In this study, actuation-step size is determined through convergent testing. In order to assure an equilibrium configuration the step size for mechanism creation should be larger than the equilibrium step. The step ratio mechanism to equilibrium (lengthening to shortening) is set to 3 . Consequently, during unfolding non-coplanar x-cable step should be 3 times larger than layer cable step (ratio inversed for folding). This value is affected by the topology of the module. Finally, the actuation step also affects the folded length. The folded length obtained by the numerical study is larger compared with the folded length predicted from the geometrical analysis due to the influence of dead load. Applying different deployment steps for each cable taking into account dead load deflection during the deployment may lead to a more effective deployment.

\section{Conclusions}

The deployment of a pentagonal tensegrity-ring module, a viable system for a footbridge application, is analyzed in this paper. The number of actuators depends on the deployment path chosen and the actuation strategy applied. The geometrical study identified the deployment-path space for contact-free deployment. Starting with a fully cable-actuated module configuration with 30 actuators (110 for the entire bridge) the number of actuators for a single module is reduced to 15 (40 for the entire bridge). The deployment path of a single module with 15 actuated cables and the use of continuous cables was validated experimentally. The deployment path with minimum actuation was analyzed using a dynamic-relaxation algorithm. The algorithm applies cable-length changes to create finite mechanisms, thus allowing the module to change length and to find new equilibrium configurations. Actuation-step size is an important parameter for a successful deployment. Stresses during deployment remain lower than in-service value. Moreover, loading direction in the tensegrity system is preserved avoiding slack cables or struts in tension. Consequently, such carefully controlled deployment is not a critical phase for member-size design of this deployable tensegrity footbridge. 


\section{Acknowledgements}

Authors would like to thank the Swiss National Science Foundation for supporting this work through contract number 200020-121552/1.

\section{Appendix}

Table 2 presents the node connectivity for a single pentagonal tensegrity-ring module.

\section{References}

Adam, B., and Smith, I. F. C. "A biomimetic structure." CISBAT 2007: Innovation in the Built Environment, LESO-PB, EPFL, 2007.

Adam, B., and Smith, I. F. C. (2007b). "Self-Diagnosis and Self-Repair of an Active Tensegrity Structure." Journal of Structural Engineering, 133(12), 1752-1761.

Adam, B., and Smith, I. F. C. (2008). "Reinforcement Learning for Structural Control." Journal of Computing in Civil Engineering, 22(2), 133-139.

Aldrich, J. B., and Skelton, R. E. "Control synthesis for a class of light and agile robotic tensegrity structures." American Control Conference, Denver, 5245-5251.

Bel Hadj Ali, N., Rhode-Barbarigos, L., Pascual Albi, A. A., and Smith, I. F. C. (2010). "Design optimization and dynamic analysis of a tensegrity-based footbridge." Engineering Structures, 32(11), 3650-3659.

Bel Hadj Ali, N., Rhode-Barbarigos, L., and Smith, I. F. C. (2011). "Analysis of clustered tensegrity structures using a modified dynamic relaxation algorithm." International Journal of Solids and Structures, 48(5), 637-647.

Bel Hadj Ali, N., and Smith, I. F. C. (2010). "Dynamic behavior and vibration control of a tensegrity structure." International Journal of Solids and Structures, 47(9), 12851296.

Bouderbala, M., and Motro, R. (1998). "Folding tensegrity systems." IUTAM-IASS Symposium on Deployable Structures: Theory and Applications, Cambridge, UK.

d'Estrée, S. T. (2003). "Using Actuated Tensegrity Structures to Produce a Responsive Architecture." The 2003 Annual Conference of the Association for Computer Aided Design In Architecture, Indianapolis (Indiana), 85-93.

Domer, B. (2003). "Performance Enhancement of Active Structures during Service Lives," EPFL, Lausanne.

Domer, B., and Smith, I. F. C. (2005). "An Active Structure that Learns." Journal of Computing in Civil Engineering, 19(1), 16-24.

Fest, E. (2002). "Une structure active de type tensegrité," EPFL, Lausanne.

Fest, E., Shea, K., and Smith, I. F. C. (2004). "Active Tensegrity Structure." Journal of Structural Engineering, 130(10), 1454-1465.

Furuya, H. (1992). "Concept of deployable tensegrity structures in space applications." International Journal of Space Structures, 7(2), 143-51.

Gantes, C., Connor, J. J., and Logcher, R. D. (1991). "Combining numerical analysis and engineering judgment to design deployable structures." Computers \& Structures, 40(2), 431-440.

Gantes, C. J. (2001). Deployable structures: analysis and design, WIT Press.

Gantes, C. J., Connor, J. J., Logcher, R. D., and Rosenfeld, Y. (1989). "Structural analysis and design of deployable structures." Computers \& Structures, 32(3-4), 661-669.

Graells Rovira, A., and Mirats Tur, J. M. (2009). "Control and simulation of a tensegritybased mobile robot." Robotics and Autonomous Systems, 57(5), 526-535. 
Hanaor, A. (1993). "Double-layer tensegrity grids as deployable structures." International Journal of Space Structures, 8, 135-45.

Ingber, D. E. (1998). "The architecture of life." Scientific American, 278(1), 48-57.

Le Saux, C., Cavaer, F., and Motro, R. (2004). "Numerical modeling of tensegrity system folding: problem of collisions between slender bars." IASS Symposium: Shell and Spatial Structures From Models to Realization, Montpellier, France.

Moored, K. W., and Bart-Smith, H. (2009). "Investigation of clustered actuation in tensegrity structures." International Journal of Solids and Structures, 46(17), 3272-3281.

Motro, R. (2005). Tenségrité, Hermes Science.

Motro, R., Maurin, B., and Silvestri, C. "Tensegrity Rings and the Hollow Rope." IASS Symposium 2006, New Olympics, New Shells and Spatial Structures, Beijing, 470-471.

Nguyen, D. A. (2009). "Etude de comportement mécanique et du pliage d'un anneau de tenségrité à base pentagonale," Université de Montpellier II, Montpellier.

Pellegrino, S. (2001). Deployable structures, Springer.

Pinaud, J.-P., Solari, S., and Skelton, R. E. "Deployment of a class 2 tensegrity boom." Smart Structures and Materials 2004: Smart Structures and Integrated Systems, San Diego, CA, USA, 155-162.

Pugh, A. (1976). An Introduction to Tensegrity, University of California Press.

Rhode-Barbarigos, L., Bel Hadj Ali, N., Motro, R., and Smith, I. F. C. (2010a). "Designing tensegrity modules for pedestrian bridges." Engineering Structures, 32(4), 1158-1167.

Rhode-Barbarigos, L., Jain, H., Kripakaran, P., and Smith, I. (2010b). "Design of tensegrity structures using parametric analysis and stochastic search." Engineering with Computers, 26(2), 193-203.

Schenk, M., Guest, S. D., and Herder, J. L. (2007). "Zero stiffness tensegrity structures." International Journal of Solids and Structures, 44(20), 6569-6583.

Skelton, R., Helton, W., Adhikari, R., Pinaud, J.-P., and Chan, W. (2009). "An Introduction to the Mechanics of Tensegrity Structures." The Mechanical Systems Design Handbook, CRC Press.

Skelton, R. E., and de Oliveira, M. C. (2009). Tensegrity systems, Springer.

Smaili, A., and Motro, R. (2005). "Folding/unfolding of tensegrity systems by removal of self-stress." HAL - CCSD.

Sultan, C. (2009). "Tensegrity motion control using internal mechanisms." 18th IEEE International Conference on Control Applications, Saint Petersburg, Russia.

Sultan, C., Corless, M., and Skelton, R. E. (2002). "Symmetrical reconfiguration of tensegrity structures." International Journal of Solids and Structures, 39(8), 2215-2234.

Sultan, C., and Skelton, R. (2003). "Deployment of tensegrity structures." International Journal of Solids and Structures, 40(18), 4637-4657.

Tan, G. E. B., and Pellegrino, S. (2008). "Nonlinear vibration of cable-stiffened pantographic deployable structures." Journal of Sound and Vibration, 314(3-5), 783-802.

Tibert, A. G. (2002). "Deployable tensegrity structures for space applications," Royal Institute of Technology, Stockholm.

Tibert, A. G., Pellegrino, S. "Deployable Tensegrity Masts." 44th AIAA/ASME/ASCE/AHS/ASC Structures, Structural Dynamics, and Material Conference and Exhibit, Norfolk, VA.

Volokh, K. Y., Vilnay, O., and Belsky, M. (2000). "Tensegrity architecture explains linear stiffening and predicts softening of living cells." Journal of Biomechanics, 33(12), 1543-1549. 


\section{List of Figure Captions}

Figure 1: Illustration of the four module tensegrity system for the deployable footbridge

Figure 2: Module topology: a) layer cables and diagonal struts, b) intermediate paired strut for a single side, c) the entire strut circuit, d) $\mathrm{x}$-cables for a single side, e) all cables of the module, $\mathrm{f}$ ) the pentagonal ring module Figure 3: Parameters describing the geometry of the ring module: $L, R$ and $\theta$

Figure 4: Deployment-path space and the path with minimum risk of strut contact for the $400 \mathrm{~cm}$ bridge module. Isometric curves corresponding to strut-to-strut distance vary from 5 (inner curve) to $45 \mathrm{~cm}$ (outer curve)

Figure 5: The small-scale physical model and its corresponding theoretical deployment-path space and experimental path. Isometric curves corresponding to strut-to-strut distance vary from 1 (inner curve) to $9 \mathrm{~cm}$ (outer curve)

Figure 6: Deployment paths with minimum strut-contact risk and minimum number of actuators for the $400 \mathrm{~cm}$ bridge module

Figure 7: The tensegrity-ring physical model with both continuous and non-actuated nylon cables when folded and unfolded

Figure 8: The deployment-analysis algorithm and its three parts: creation of mechanism, new equilibrium and constraint checking

Figure 9: The deployment path space and the path obtained by the deployment analysis algorithm for the $400 \mathrm{~cm}$ bridge module

Figure 10: Internal forces for actuated layer cables, actuated x-cables, struts and non-actuated x-cables throughout the deployment for a single bridge module 


\section{Tables}

Table 1: Nodal coordinates of the pentagonal ring module

\begin{tabular}{lll}
\hline Node $\mathrm{n}^{\circ}$ & Cylindrical coordinates & Cartesian coordinates \\
\hline 1 & $0,0, \mathrm{R}$ & $0,0, \mathrm{R}$ \\
2 & $0,2 \pi / 5, \mathrm{R}$ & $0, \mathrm{R} \sin (2 \pi / 5), \mathrm{R} \cos (2 \pi / 5)$ \\
3 & $0,4 \pi / 5, \mathrm{R}$ & $0, \mathrm{R} \sin (4 \pi / 5), \mathrm{R} \cos (4 \pi / 5)$ \\
4 & $0,6 \pi / 5, \mathrm{R}$ & $0, \mathrm{R} \sin (6 \pi / 5), \mathrm{R} \cos (6 \pi / 5)$ \\
5 & $0,8 \pi / 5, \mathrm{R}$ & $0, \mathrm{R} \sin (8 \pi / 5), \mathrm{R} \cos (8 \pi / 5)$ \\
6 & $\mathrm{~L} / 2, \pi+\theta / 2, \mathrm{R}$ & $\mathrm{L} / 2, \mathrm{R} \sin (\pi+\theta / 2), \mathrm{R} \cos (\pi+\theta / 2)$ \\
7 & $\mathrm{~L} / 2,7 \pi / 5+\theta / 2, \mathrm{R}$ & $\mathrm{L} / 2, \mathrm{R} \sin (7 \pi / 5+\theta / 2), \mathrm{R} \cos (7 \pi / 5+\theta / 2)$ \\
8 & $\mathrm{~L} / 2,9 \pi / 5+\theta / 2, \mathrm{R}$ & $\mathrm{L} / 2, \mathrm{R} \sin (9 \pi / 5+\theta / 2), \mathrm{R} \cos (9 \pi / 5+\theta / 2)$ \\
9 & $\mathrm{~L} / 2, \pi / 5+\theta / 2, \mathrm{R}$ & $\mathrm{L} / 2, \mathrm{R} \sin (\pi / 5+\theta / 2), \mathrm{R} \cos (\pi / 5+\theta / 2)$ \\
10 & $\mathrm{~L} / 2,3 \pi / 5+\theta / 2, \mathrm{R}$ & $\mathrm{L} / 2, \mathrm{R} \sin (3 \pi / 5+\theta / 2), \mathrm{R} \cos (3 \pi / 5+\theta / 2)$ \\
11 & $\mathrm{~L}, \theta, \mathrm{R}$ & $\mathrm{L}, \mathrm{R} \sin (\theta), \mathrm{R} \cos (\theta)$ \\
12 & $\mathrm{~L}, 2 \pi / 5+\theta, \mathrm{R}$ & $\mathrm{L}, \mathrm{R} \sin (2 \pi / 5+\theta), \mathrm{R} \cos (2 \pi / 5+\theta)$ \\
13 & $\mathrm{~L}, 4 \pi / 5+\theta, \mathrm{R}$ & $\mathrm{L}, \mathrm{R} \sin (4 \pi / 5+\theta), \mathrm{R} \cos (4 \pi / 5+\theta)$ \\
14 & $\mathrm{~L}, 6 \pi / 5+\theta, \mathrm{R}$ & $\mathrm{L}, \mathrm{R} \sin (6 \pi / 5+\theta), \mathrm{R} \cos (6 \pi / 5+\theta)$ \\
15 & $\mathrm{~L}, 8 \pi / 5+\theta, \mathrm{R}$ & $\mathrm{L}, \mathrm{R} \sin (8 \pi / 5+\theta), \mathrm{R} \cos (8 \pi / 5+\theta)$ \\
\hline
\end{tabular}


Table 2: Node connectivity and element description for the pentagonal ring module

\begin{tabular}{|c|c|c|c|}
\hline Element & Node & Description & Description \\
\hline 1 & 1 & 12 & Diagonal strut \\
\hline 2 & 2 & 13 & Diagonal strut \\
\hline 3 & 3 & 14 & Diagonal strut \\
\hline 4 & 4 & 15 & Diagonal strut \\
\hline 5 & 5 & 11 & Diagonal strut \\
\hline 6 & 1 & 9 & Intermediate strut \\
\hline 7 & 2 & 10 & Intermediate strut \\
\hline 8 & 3 & 6 & Intermediate strut \\
\hline 9 & 4 & 7 & Intermediate strut \\
\hline 10 & 5 & 8 & Intermediate strut \\
\hline 11 & 11 & 7 & Intermediate strut \\
\hline 12 & 12 & 8 & Intermediate strut \\
\hline 13 & 13 & 9 & Intermediate strut \\
\hline 14 & 14 & 10 & Intermediate strut \\
\hline 15 & 15 & 6 & Intermediate strut \\
\hline 16 & 1 & 2 & Layer cable \\
\hline 17 & 2 & 3 & Layer cable \\
\hline 18 & 3 & 4 & Layer cable \\
\hline 19 & 4 & 5 & Layer cable \\
\hline 20 & 5 & 1 & Layer cable \\
\hline 21 & 11 & 12 & Layer cable \\
\hline 22 & 12 & 13 & Layer cable \\
\hline 23 & 13 & 14 & Layer cable \\
\hline 24 & 14 & 15 & Layer cable \\
\hline 25 & 15 & 11 & Layer cable \\
\hline 26 & 6 & 1 & X-cable \\
\hline 27 & 6 & 2 & X-cable \\
\hline 28 & 6 & 11 & X-cable \\
\hline 29 & 6 & 12 & X-cable \\
\hline 30 & 7 & 2 & X-cable \\
\hline 31 & 7 & 3 & X-cable \\
\hline 32 & 7 & 12 & X-cable \\
\hline 33 & 7 & 13 & X-cable \\
\hline 34 & 8 & 3 & X-cable \\
\hline 35 & 8 & 4 & X-cable \\
\hline 36 & 8 & 13 & X-cable \\
\hline 37 & 8 & 14 & X-cable \\
\hline
\end{tabular}




\begin{tabular}{llll}
38 & 9 & 4 & X-cable \\
39 & 9 & 5 & X-cable \\
40 & 9 & 14 & X-cable \\
41 & 9 & 15 & X-cable \\
42 & 10 & 1 & X-cable \\
43 & 10 & 5 & X-cable \\
44 & 10 & 11 & X-cable \\
45 & 10 & 15 & X-cable \\
\hline
\end{tabular}




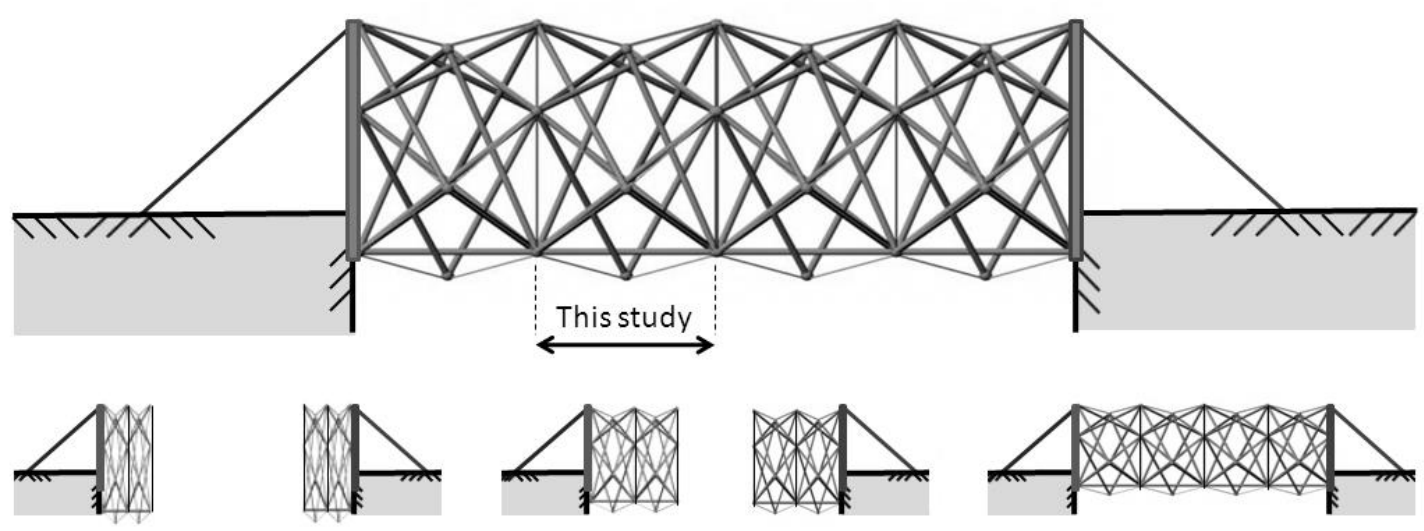

Figure 11: Illustration of the four module tensegrity system for the deployable footbridge 


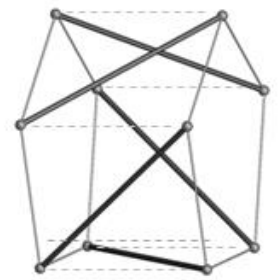

a)

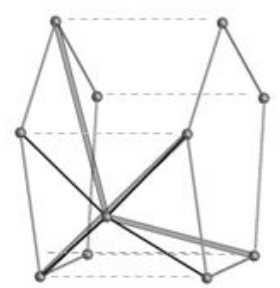

d)

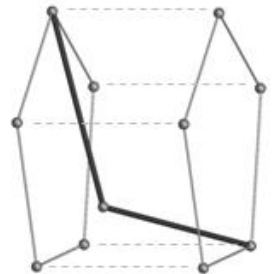

b)

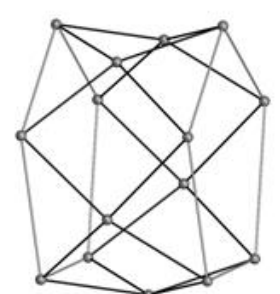

e)

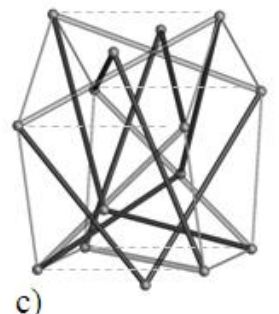

c)

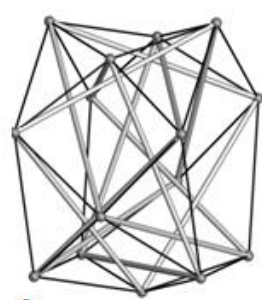

f)

Figure 12: Module topology: a) layer cables and diagonal struts, b) intermediate paired strut for a single side, c) the entire strut circuit, $\mathrm{d}$ ) $\mathrm{x}$-cables for a single side, e) all cables of the module, $\mathrm{f}$ ) the pentagonal ring module 

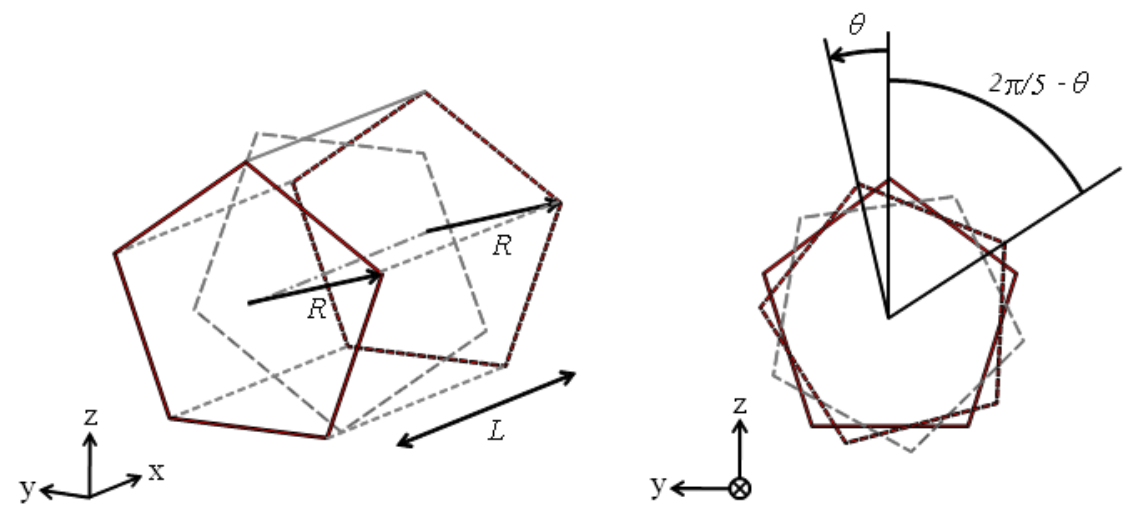

Figure 13: Parameters describing the geometry of the ring module: $L, R$ and $\theta$ 
Transverse rotation $\theta$ [rad]

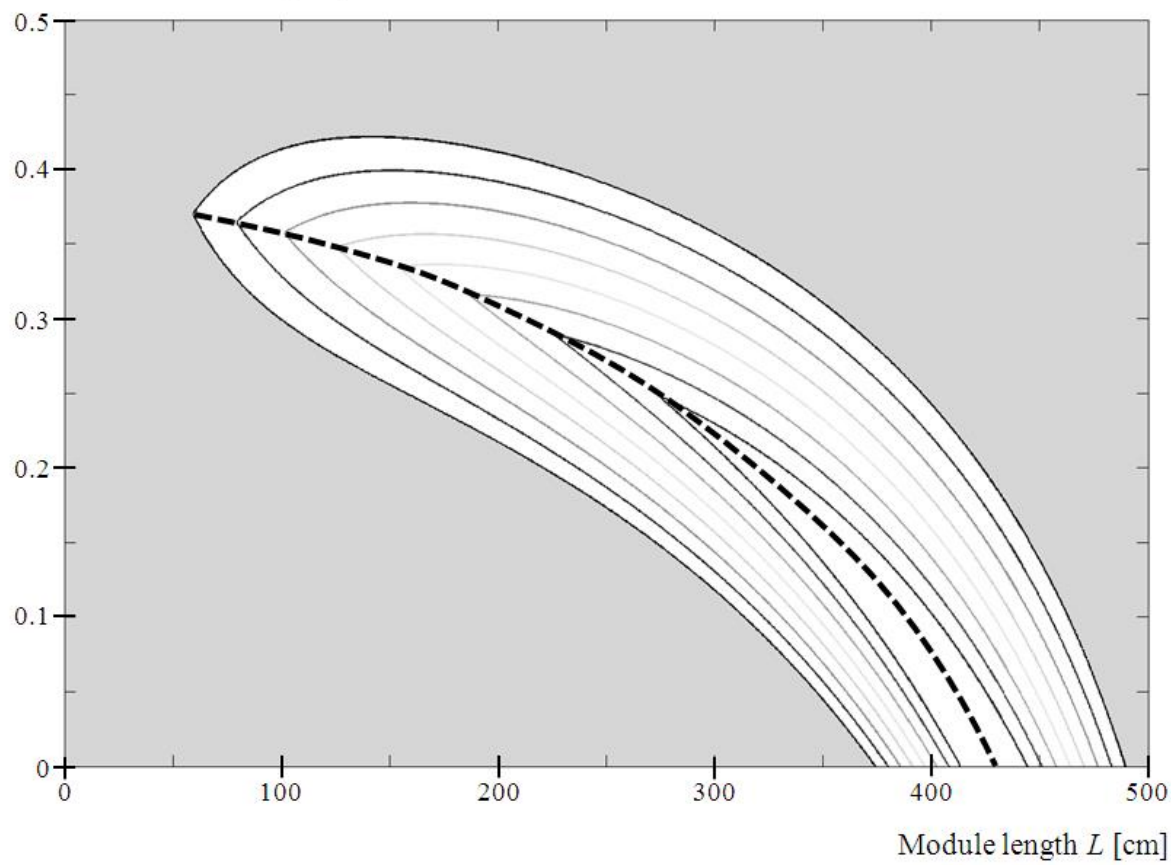

Figure 14: Deployment-path space and the path with minimum risk of strut contact for the $400 \mathrm{~cm}$ bridge module. Isometric curves corresponding to strut-to-strut distance vary from 5 (inner curve) to $45 \mathrm{~cm}$ (outer curve) 

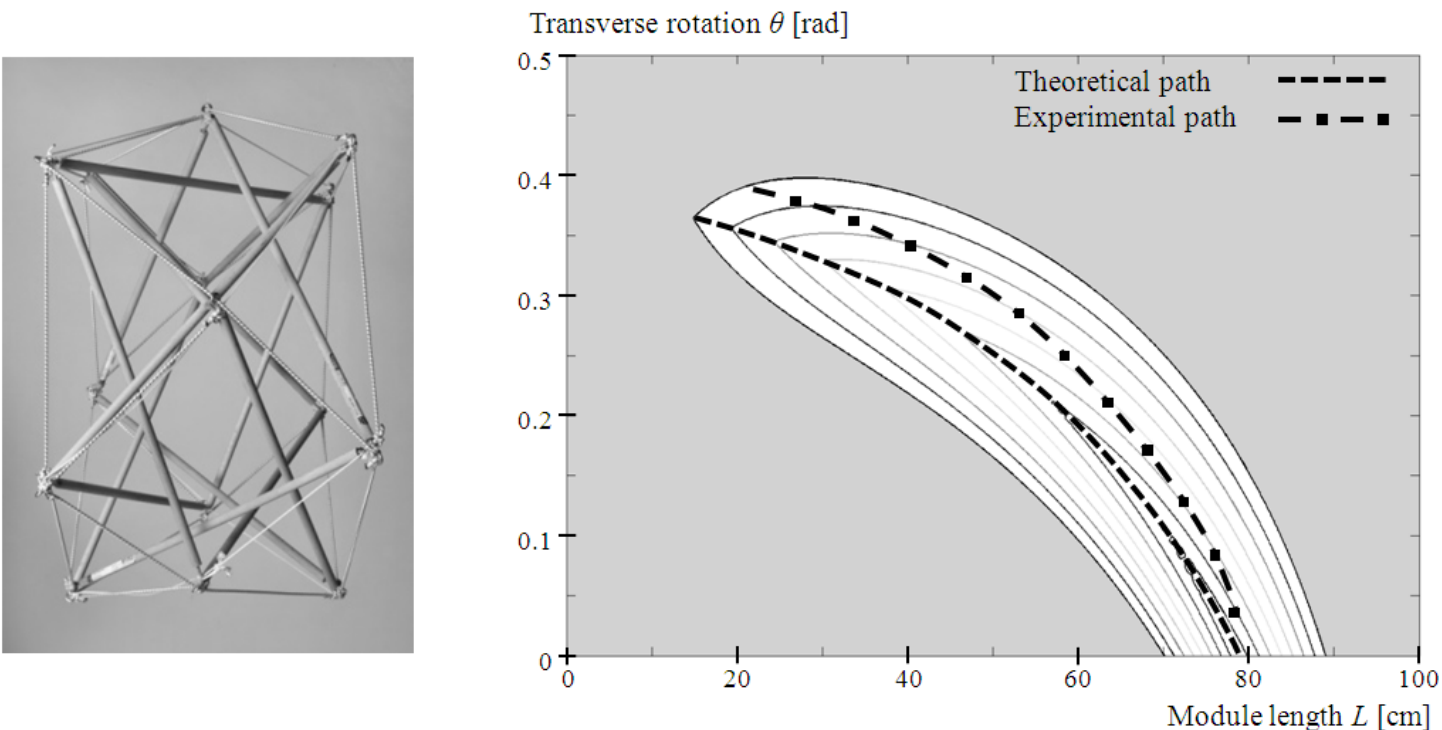

Figure 15: The small-scale physical model and its corresponding theoretical deployment-path space and experimental path. Isometric curves corresponding to strut-to-strut distance vary from 1 (inner curve) to $9 \mathrm{~cm}$ (outer curve) 


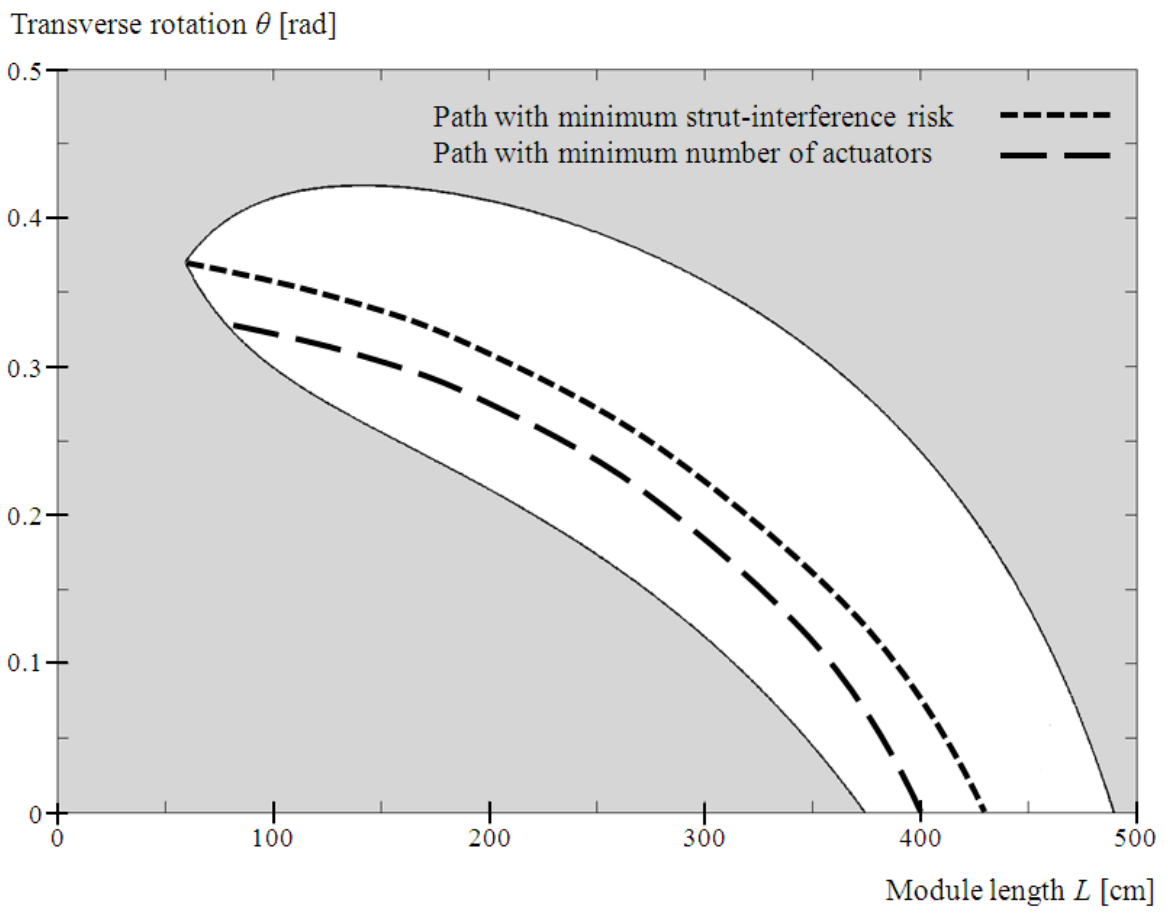

Figure 16: Deployment paths with minimum strut-contact risk and minimum number of actuators for the $400 \mathrm{~cm}$ bridge module 


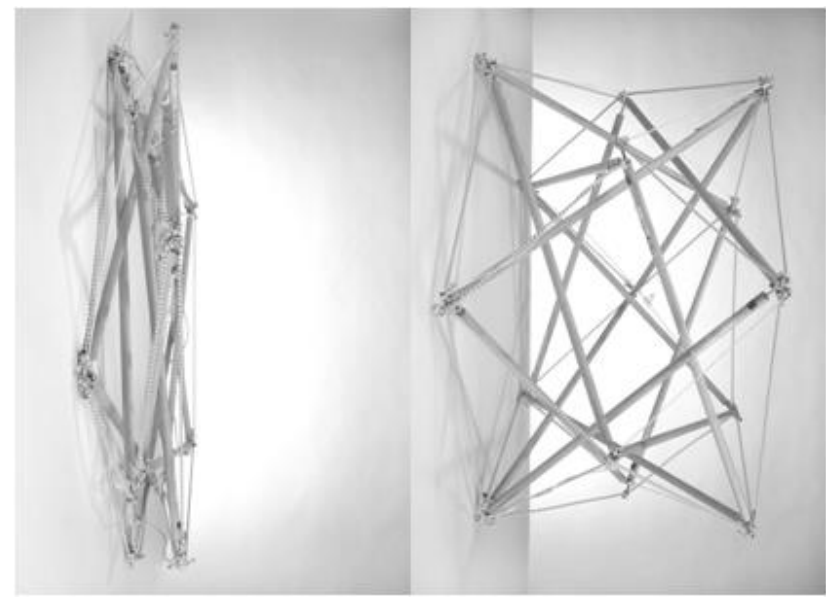

Figure 17: The tensegrity-ring physical model with both continuous and non-actuated nylon cables when folded and unfolded 


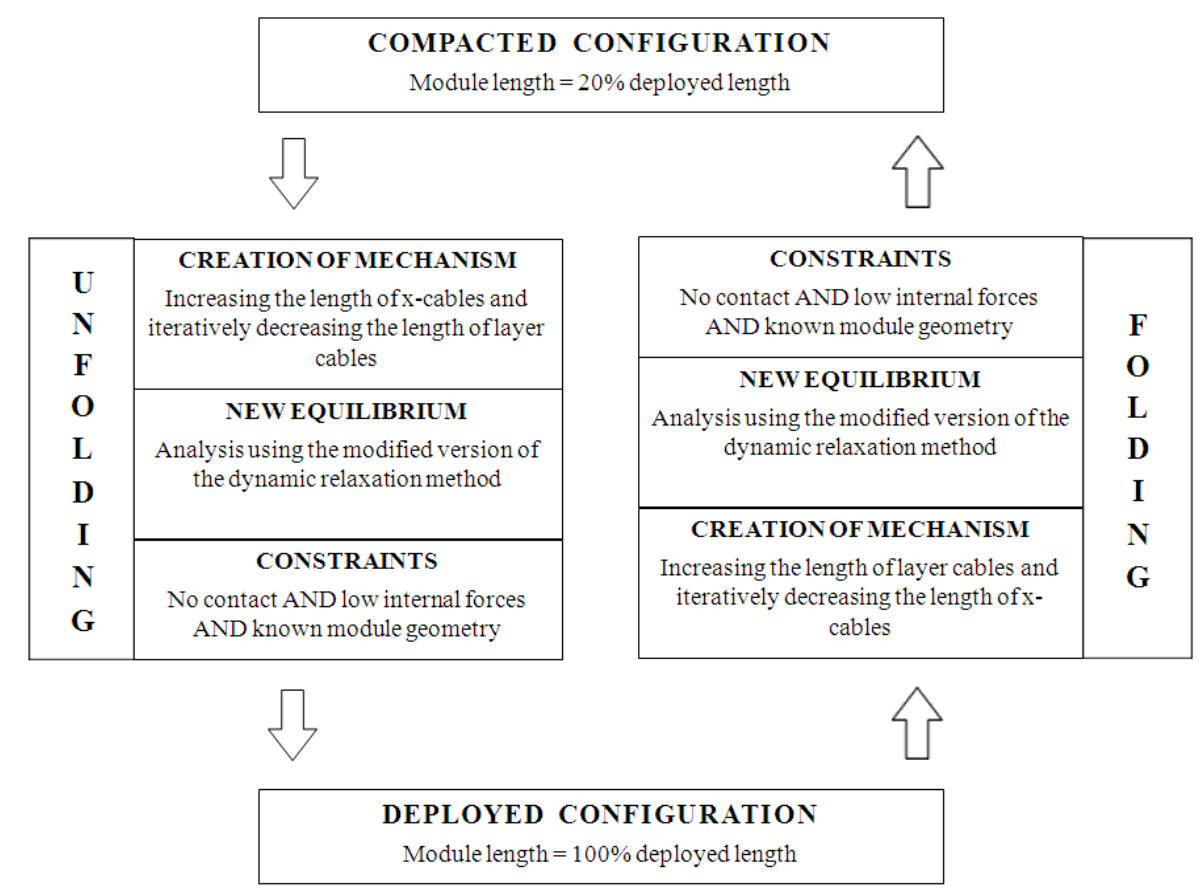

Figure 18: The deployment-analysis algorithm and its three parts: creation of mechanism, new equilibrium and constraint checking 


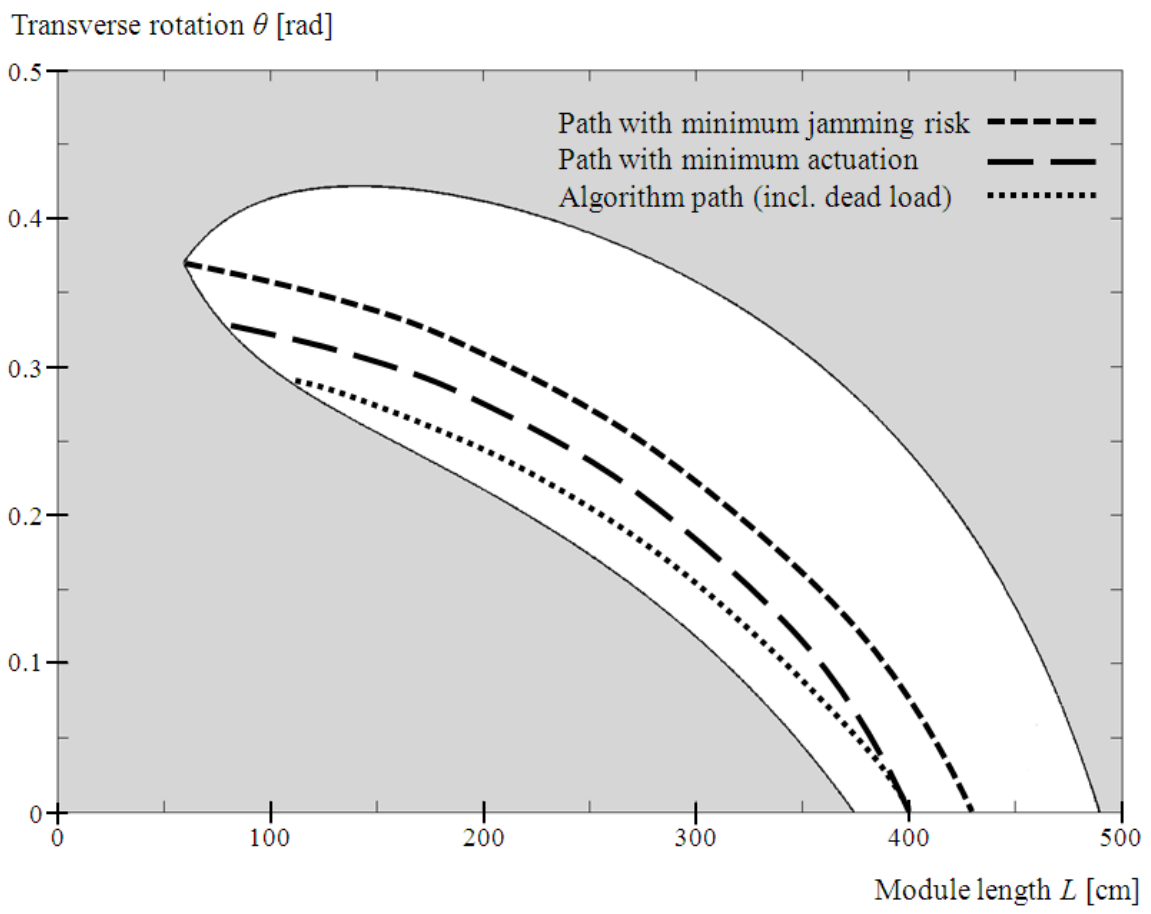

Figure 19: The deployment path space and the path obtained by the deployment analysis algorithm for the 400 $\mathrm{cm}$ bridge module 
Internal force $[\mathrm{kN}]$

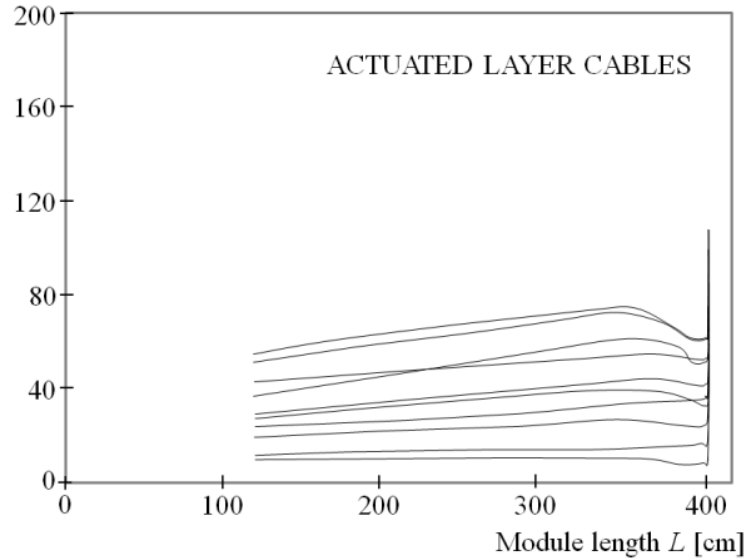

Internal force $[\mathrm{kN}]$

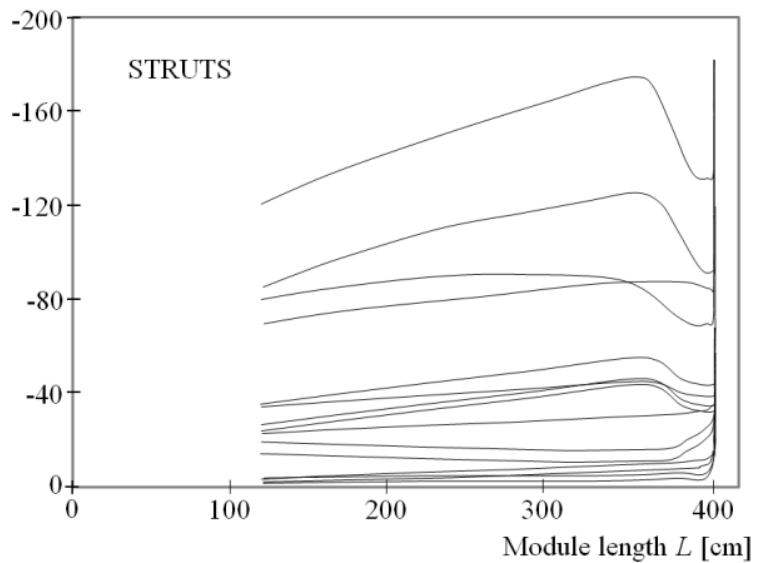

Internal force $[\mathrm{kN}]$

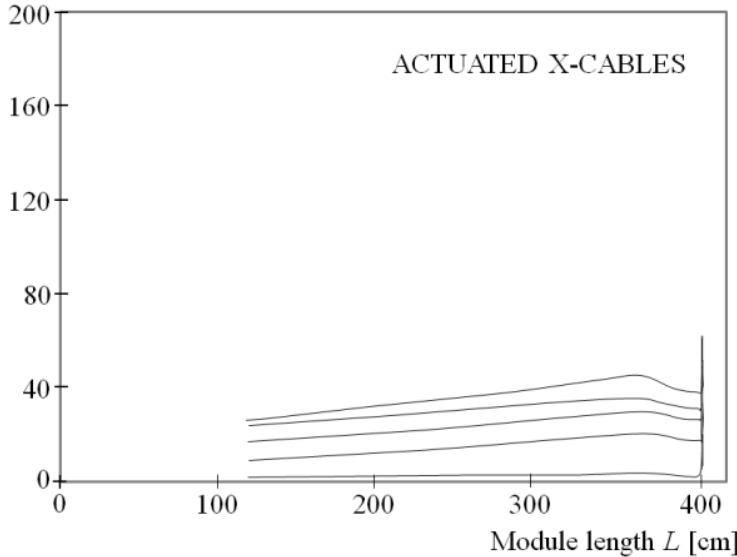

Internal force $[\mathrm{kN}]$

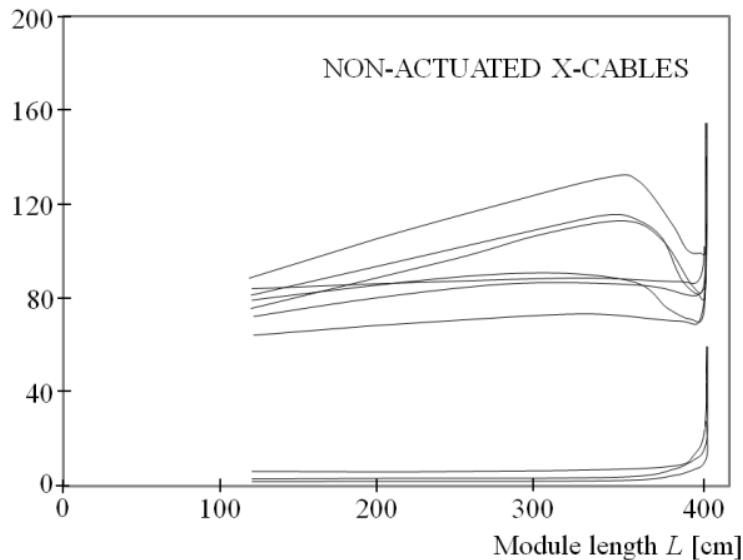

Figure 20: Internal forces for actuated layer cables, actuated x-cables, struts and non-actuated x-cables throughout the deployment for a single bridge module 